Paper

\title{
Control of transportation trails by distributed autonomous agents inspired by the foraging behavior of ants
}

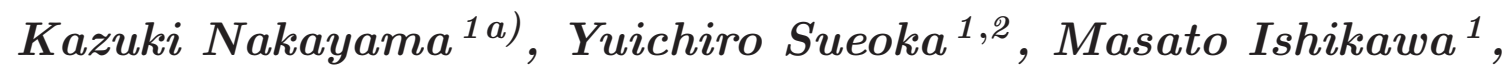 \\ Yasuhiro Sugimoto $^{1}$, and Koichi Osuka ${ }^{1}$ \\ ${ }^{1}$ Department of Mechanical Engineering, Osaka University \\ 2-1 Yamada-oka, Suita, Osaka 565-0871, Japan \\ ${ }^{2}$ Research Fellow of Japan Society for the Promotion of Science \\ 5-3-1 Koji-machi, Chiyoda-ku, Tokyo 102-0083, Japan \\ a)nakayama@dsc.mech.eng.osaka-u.ac.jp
}

Received January 24, 2014; Revised May 5, 2014; Published October 1, 2014

\begin{abstract}
In this paper, we discuss some phenomena of distributed transportation inspired by the foraging behavior of ants, in the light of space-discretization (or cellular automata) approach. We define fundamental event rules in a cellular world, and introduce two types of ant agents: one is an oriented ant, which perceives pheromones; the other is a random ant, which does not perceive pheromones. We then take a closer look at the number of pheromone trails formed by homogeneous and heterogeneous agents. Moreover, we propose a distributed control method for the number of formed pheromone trails, and examine the effectiveness of the proposed method in several simulations.
\end{abstract}

Key Words: distributed autonomous system, heterogeneous agents, cellular automata

\section{Introduction}

Living things in nature select their own behavior adaptively in an unknown environment in real time. Generally, this is done not only by human beings, whose brains consist of more than one hundred billion neurons, but also by insects, whose brains consists of no more than one million neurons [1]. In particular, a colony of social insects like ants, termites or bees generate global patterns, which are flexible and adaptive, although each individual decides its actions in accordance with very simple rules based on local interactions [2]. Here we discuss the foraging behavior of ants as an example. The colony of ants can form a huge trail between their nest and food sources, whose length has been known to reach over one kilometer. It appears that ants decide their own actions according to complicated behavior rules, based on global information or a centralized control system. In fact, however, ants act based on only simple behavior rules, which stem from local information, such as pheromones emitted by other ants $[3,4]$. Thus, the analysis of phenomena such as stable pheromone trails, which are flexible and adaptive, is likely to yield many useful ideas for system planning. 


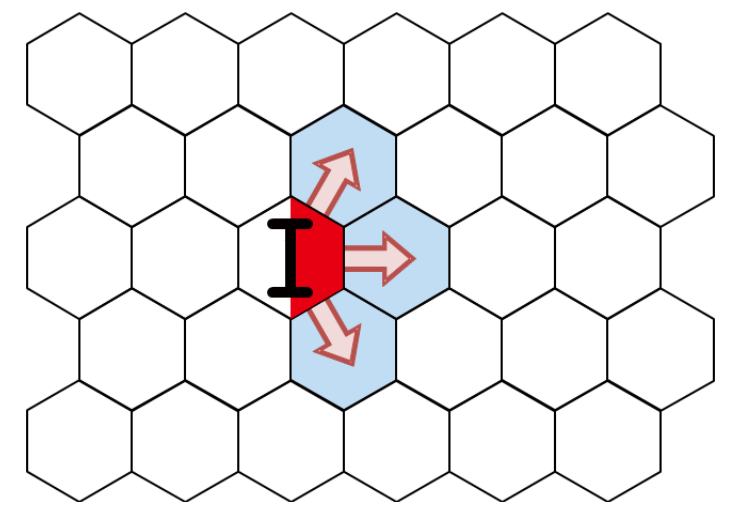

Fig. 1. The ant model in hexagonal grid space: decision of the direction to follow pheromones.

Thus far, foraging behavior of ants has been investigated from the point of view of entomology [3-5]. The results have been applied to engineering, in particular in swarm robotics [6-10] and optimization algorithms $[11,12]$. Our aim, however, is at understanding general mechanisms behind "global spatial pattern formed by locally perceiving/acting agents" using sufficiently simple model, inspired by foraging behavior of social insects. In particular, we focus on analyses of distributed transportation by the colony of autonomous agents with heterogeneous or autonomous switching rules. In this research, we use cellular automata approach to analyze global pattern generation. This cellular automata approach was first proposed by Stephen Wolfram to investigate complex systems [13]. These detailed analyses lead to an understanding of the many complex systems generated by agents [14].

In this research, we suppose a tessellation of the 2-dimensional Euclidean space with unit regular hexagons, as shown in Fig. $1[15,16]$. We define fundamental event rules in this cellular world and construct a discrete version of the distributed transportation model inspired by ant foraging behavior. We then examine the distributed transportation by autonomous agents using pheromone trails. The organization of the present paper is as follows. In Section 2, we build a discrete event model of distributed transportation inspired by the foraging behavior of ants. In construction of ant agents, we assume that they have two modes: exploration and transportation, and four action rules in a discretized environment. Moreover, we propose two types of ant agents: one is a random ant, which follows only one action rule and the other is an oriented ant, which follows all action rules. Furthermore, we construct a discretized model of the dynamics of pheromones which ants emit and perceive. In Section 3, we analyze distributed transportation within the colony of homogeneous ants and that of heterogeneous ants by focusing on two numerical indices: the number of pheromone trails formed between the ants' nest and food sources, and the ratio of object transportation (the number of food packets transported to their nest per step). In Section 4, from the analyses of distributed transportation by heterogeneous agents, we propose a distributed method to control the number of pheromone trails formed between the nest and food sources, using switching agents which switch between two types of behavior procedures autonomously, and examine the effectiveness of the proposed method by several simulations.

\section{Discrete model of distributed transportation inspired by ant forag- ing behavior}

In this section, we construct a discrete version of distributed transportation inspired by the foraging behavior of ants.

\subsection{The switching model between exploration and transportation modes}

Every ant has two modes: exploration and transportation. Exploration mode is the state from leaving the nest to picking up a food packet at the food source, and transportation mode is the state from picking up a food packet to dropping it off at the nest (see Fig. 2). 


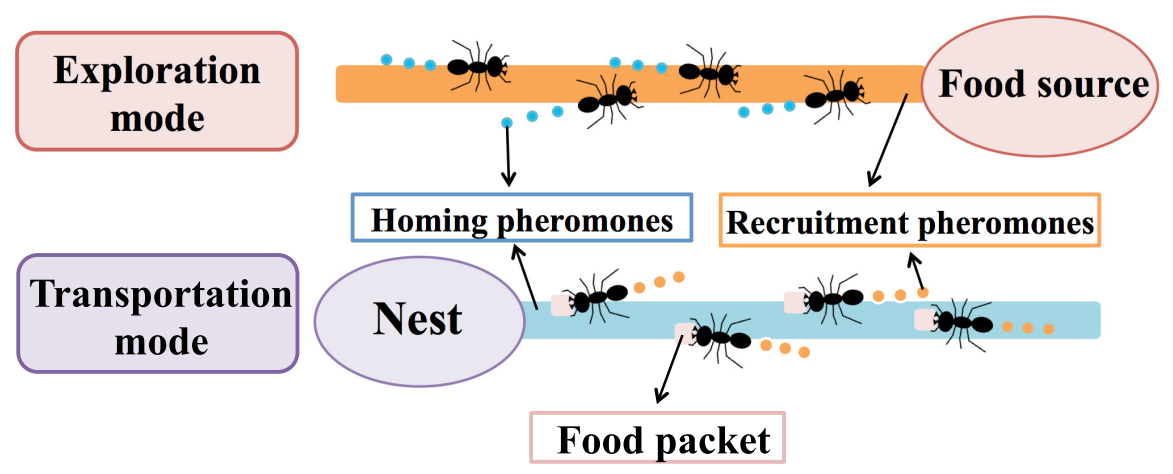

Fig. 2. The switching model between exploration and transportation modes in the foraging behavior of ants.

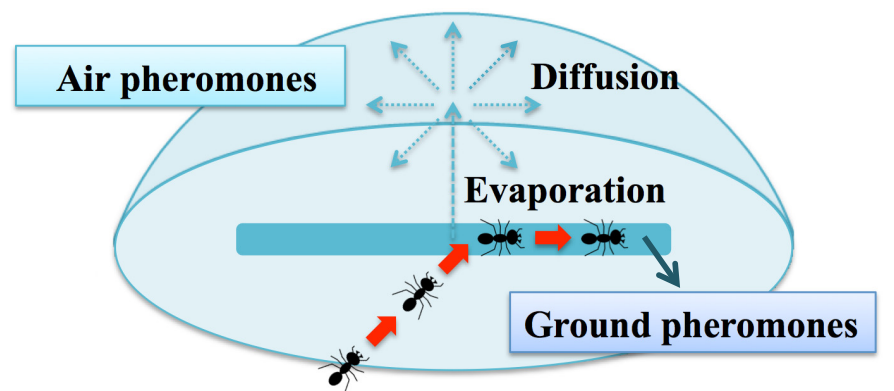

Fig. 3. Evaporation and diffusion of pheromones.

\section{Mode 1 (Exploration mode)}

- Ants emit homing pheromones at every step.

- Ants perceive recruitment pheromones.

- Ants switch to transportation mode as they pick up a food packet at the food source.

\section{Mode 2 (Transportation mode)}

- Ants emit recruitment pheromones at every step.

- Ants perceive homing pheromones.

- Ants switch to exploration mode as they lay the food packet at their nest.

Every food packet is transported by one ant, and food at the source is never exhausted. Ants transport a food packet from a food source to their nest through emitting and perceiving different pheromones in exploration or transportation modes.

\subsection{The dynamics of pheromones and their discretized model}

In this section, we construct a discrete version of pheromone model based on the natural ones emitted by real ants. As mentioned in the last subsection, ants emit two types of pheromones: homing and recruitment pheromones. Both pheromones are volatile, so they evaporate from the field, and diffuse into space, as shown in Fig. 3. We separate the pheromones into ground and air ones.

We define the chemical concentration of the pheromones, $P_{j}(x, y, z, t)$, where $(x, y, z) \in \mathbb{R}^{3}$ are the coordinates of the pheromones, and $t \geq 0$ is time. In addition, $j \in\{1,2\}$ are the indices of the two types of pheromones, where $j=1$ and $j=2$ denote homing and recruitment pheromones, respectively. Then $P_{j}(x, y, z, t)$ is the concentration of the ground pheromones if $z=0$, and that of the air pheromones if $z>0$. It is known that evaporation and diffusion of pheromones are determined by the following Eqs. [17, 18]:

$$
\frac{\partial}{\partial t} P_{j}(x, y, z, t)= \begin{cases}-\gamma_{e v a_{j}} P_{j}(x, y, z, t)+\gamma_{a d d_{j}} N_{j}(x, y, t) & (z=0) \\ \gamma_{d i f_{j}} \nabla^{2} P_{j}(x, y, z, t)+\gamma_{e v a_{j}} P_{j}(x, y, 0, t) & (z>0)\end{cases}
$$


where $\gamma_{e v a_{j}}$ denotes the evaporation coefficient of pheromones $j, \gamma_{d i f_{j}}$ denotes the diffusion coefficient of pheromones $j, \gamma_{a d d_{j}}$ denotes the amount of pheromones $j$ emitted by one ant at every step, and $N_{j}(x, y, t)$ denotes the total number of ants in mode $j$ at $(x, y)$ at time $t$. In this paper, we introduce hexagonal tessellation of the plane, considering its advantage on its inherit symmetry with respect to rotations, such as the fact that a cell is equally distant from all the neighboring cells. This property is well suitable for defining discrete version of diffusion dynamics of the pheromones. The discretized model of the dynamics of pheromones is derived from discretizing Eq. (1) on the hexagonal grid as follows. In the diffusion phenomena of pheromones, we suppose that the ground $(z=0)$ is a reflecting boundary and the others are absorbing ones.

$$
\begin{aligned}
& P_{j}\left.n_{x}, n_{y}, n_{z}, \tau+1\right) \\
& \quad= \begin{cases}\left(1-\gamma_{e v a_{j}}\right) P_{j}\left(n_{x}, n_{y}, n_{z}, \tau\right)+\gamma_{a d d_{j}} N_{j}\left(n_{x}, n_{y}, \tau\right) & \left(n_{z}=0\right) \\
\left(1-7 \gamma_{d i f_{j}}\right) P_{j}\left(n_{x}, n_{y}, n_{z}, \tau\right)+P_{I N_{j}}\left(n_{x}, n_{y}, n_{z}, \tau\right)+\gamma_{\text {eva }} P_{j}\left(n_{x}, n_{y}, 0, \tau\right) & \left(n_{z}>0\right)\end{cases}
\end{aligned}
$$

where $n_{x}, n_{y} \in \mathbb{Z}$ and $n_{z} \in\{0,1\}$ denotes the coordinates of the pheromones in the cellular world, and $\tau \in \mathbb{Z}^{+}$denotes time step. Also, $P_{I N_{j}}\left(n_{x}, n_{y}, n_{z}, \tau\right)$ denotes the concentration of pheromones $j$ flowing into cell $(x, y)$ from the six nearest-neighbor cells, as follows:

$$
\begin{aligned}
P_{I N_{j}}\left(n_{x}, n_{y}, n_{z}, \tau\right)= & \gamma_{d i f_{j}}\left\{P_{j}\left(n_{x}+1, n_{y}, n_{z}, \tau\right)+P_{j}\left(n_{x}+\operatorname{odd}\left(n_{y}\right), n_{y}+1, n_{z}, \tau\right)\right. \\
& +P_{j}\left(n_{x}-\operatorname{even}\left(n_{y}\right), n_{y}+1, n_{z}, \tau\right)+P_{j}\left(n_{x}-1, n_{y}, n_{z}, \tau\right) \\
& \left.+P_{j}\left(n_{x}-\operatorname{even}\left(n_{y}\right), n_{y}-1, n_{z}, \tau\right)+P_{j}\left(n_{x}+\operatorname{odd}\left(n_{y}\right), n_{y}-1, n_{z}, \tau\right)\right\}
\end{aligned}
$$

where even $(l)$ is 1 if $l$ is an even number, 0 otherwise, and odd $(l)$ is 1 if $l$ is an odd number, 0 otherwise.

Here, we define the threshold concentration of ground pheromones and that of air pheromones $j$ which ants can perceive as $G_{t h r_{j}}$ and $A_{t h r_{j}}$, respectively. Table I shows all of the above-mentioned parameter values. These parameter values are chosen from reasonable ones based on the biological references [19-22]. In this work we set the parameter values of homing and recruitment pheromones to be equal.

\subsection{Primitive action rules}

In this paper, we suppose that every ant is placed at the center of a hexagonal cell (Fig. 1), thus its position is expressed by a pair of integers which belongs to $\mathbb{Z}^{2}$. Moreover, its orientation angle on the hexagonal grid space is also discrete: it is confined to $0, \pm 1 \gamma, \pm 2 \gamma, \pm 3 \gamma, \cdots \in \mathbb{S}\left(\gamma=\frac{\pi}{3}\right)$, where $3 \gamma$ and $-3 \gamma$ are identified to each other. As a slight abuse of notation, we identify $\gamma i \in \mathbb{S}(i \in \mathbb{Z})$ with $i$ itself. In summary, the space of orientation angles is identified with the set of integers modulo 6 :

$$
\mathbb{Z}_{6}=\{0, \pm 1, \pm 2,3\}
$$

Therefore, the configuration of every ant on the hexagonal grid space is expressed by an element of $\mathbb{Z}^{2} \times \mathbb{Z}_{6}$. Also, there is no limit on the number of ants in a single cell in this paper. Namely, we do not adopt excluded volume. Furthermore, we suppose that ants perceive both ground and air pheromones on the three blue cells in Fig. 1; i.e. their front, front right and front left cells. Moreover, we assume that ants perceive whether their nest is in the direction of movement based on the navigational abilities of real ones such as a solar compass [23]. Ants autonomously choose one action rule from the following four and move in the cellular world.

Table I. Parameter values of pheromones.

\begin{tabular}{|c||l|l|}
\hline & Parameters of pheromones & Values \\
\hline \hline$\gamma_{\text {eva }}$ & Pheromone evaporation coefficient & $6.7 \times 10^{-2}$ \\
\hline$\gamma_{d i f}$ & Pheromone diffusion coefficient & $7.0 \times 10^{-2}$ \\
\hline$\gamma_{\text {add }}$ & Amount of pheromones emitted by an ant & $1.0 \times 10^{2}$ \\
\hline$G_{t h r}$ & Threshold concentration of ground pheromones & $1.0 \times 10^{-1}$ \\
\hline$A_{t h r}$ & Threshold concentration of air pheromones & $1.0 \times 10^{-4}$ \\
\hline
\end{tabular}




\section{Action rule 1 (Ground pheromone tracking)}

When ants perceive ground pheromones in their front, front right or front left cells, they follow this rule. If they perceive ground pheromones in the front, they move to that cell. If they do not perceive ground pheromones in the front cell, but perceive them in their front right or left cells, they choose one cell from the two with the following probability $p_{r 1}\left(n_{x}, n_{y}, \tau, k\right)(k \in\{-1,1\})$ and move to it. Here, $p_{r 1}\left(n_{x}, n_{y}, \tau, k\right)$ denotes the probability of that ants at $\left(n_{x}, n_{y}\right)$ choose the cell in their $k$ direction at time $\tau$. Also, their $k=-1,1$ direction denotes their front right and front left cells, respectively.

$$
p_{r 1}\left(n_{x}, n_{y}, \tau, k\right)=\frac{\exp \left\{G^{k}\left(n_{x}, n_{y}, \tau\right)\right\}}{\exp \left\{G^{-1}\left(n_{x}, n_{y}, \tau\right)\right\}+\exp \left\{G^{1}\left(n_{x}, n_{y}, \tau\right)\right\}}
$$

where $G^{k}\left(n_{x}, n_{y}, \tau\right)$ denotes the concentration of grand pheromones in the cell in the $k$ direction of ants at $\left(n_{x}, n_{y}\right)$ and time $\tau$.

\section{Action rule 2 (Air pheromone tracking)}

When ants do not perceive ground pheromones on their front, front right or front left cell, but perceive air pheromones, they follow this rule. They choose one cell out of three with probability $p_{r 2}\left(n_{x}, n_{y}, \tau, k\right)(k \in\{-1,0,1\})$ and move to it. Here, $p_{r 2}\left(n_{x}, n_{y}, \tau, k\right)$ denotes the probability that ants at $\left(n_{x}, n_{y}\right)$ choose the cell in direction $k$ at time $\tau$. Also, their $k=-1,0,1$ directions denote their front right, front and front left, respectively.

$$
p_{r 2}\left(n_{x}, n_{y}, \tau, k\right)=\frac{\exp \left\{A^{k}\left(n_{x}, n_{y}, \tau\right)\right\}}{\sum_{l=-1}^{1} \exp \left\{A^{l}\left(n_{x}, n_{y}, \tau\right)\right\}}
$$

where $A^{k}\left(n_{x}, n_{y}, \tau\right)$ denotes the concentration of air pheromones in the cell in the $k$ direction of ants at $\left(n_{x}, n_{y}\right)$ and time $\tau$.

\section{Action rule 3 (Random walk)}

When ants do not perceive either ground or air pheromones in their front, front right or front left cells, they follow this rule. They move to their front cell with probability $\varepsilon(0 \leq \varepsilon \leq 1)$ and their front right or front left cells with probability $\frac{1-\varepsilon}{2}$, respectively. In this paper, we set $\varepsilon$ to 0.8 .

\section{Action rule 4 (U-turn)}

In transportation mode only, when ants perceive that their nest does not exist in front of them, they reverse direction.

\subsection{Two types of behavior procedures}

In this work, we propose two types of ant agents based on four action rules in the section above: a random ant and an oriented ant. Each ant agent is defined below. Also, in each mode (i.e., exploration and transportation) each ant agent follows action rules with priority shown by the arrows.

\section{Procedure Type 1 (Random)}

Random ants do not perceive pheromones and follow only action rule 3 (random walk). Thus they always move randomly, emitting pheromones without tracking pheromones.

- Exploration mode: action rule 3

- Transportation mode: action rule 3

\section{Procedure Type 2 (Oriented)}

Oriented ants perceive pheromones and follow one of four action rules with priority shown by the arrows below. Thus they move stochastically to the cell whose concentration of pheromones is high.

- Exploration mode: Action rule priority $1 \rightarrow 2 \rightarrow 3$

- Transportation mode: Action rule priority $4 \rightarrow 1 \rightarrow 2 \rightarrow 3$ 


\section{Effect of mixing heterogeneous behavior procedures in distributed transportation}

In this section, we analyze the distributed transportation by ant agents with the proposed discrete models. Suppose the field surrounded by an outer boundary wall contains $107 \times 107$ cells; the ants' nest is placed in the center of the field (orange area in Fig. 4); six food sources are also placed in the field (six blue areas in Fig. 4). In Fig. 4, the nest and the food sources are visualized as multiple cells just to improve their visibility. In simulations, they consist of a single cell that is center of multiple cells in Fig. 4. The initial state of the field is shown in Fig. 4 at the zeroth time step. We then analyze the distributed transportation focusing on two evaluation indices: the number of stable pheromone trails formed between the ants' nest and food sources, and the ratio of object transportation (the number of food packets transported to the nest per step). In this paper, we define "a stable pheromone trail" as a pheromone trail that connects a food source to the nest, and remains unchanged unless the food source runs out. In other words, it is "an invariant pheromone trail", because we suppose that a food at the source is never exhausted in this paper.

\subsection{Distributed transportation by a colony of homogeneous agents}

Let us begin to discuss the distributed transportation by the colony of homogeneous agents (oriented ants only). Figure 4 shows the snapshots of the distributed transportation by the colony of 600 oriented ants. Pheromone trails are gradually formed between ants' nest and food sources by oriented ants, and most of ants follow two stable pheromone trails for about 20000 steps. Figure 5(a) shows
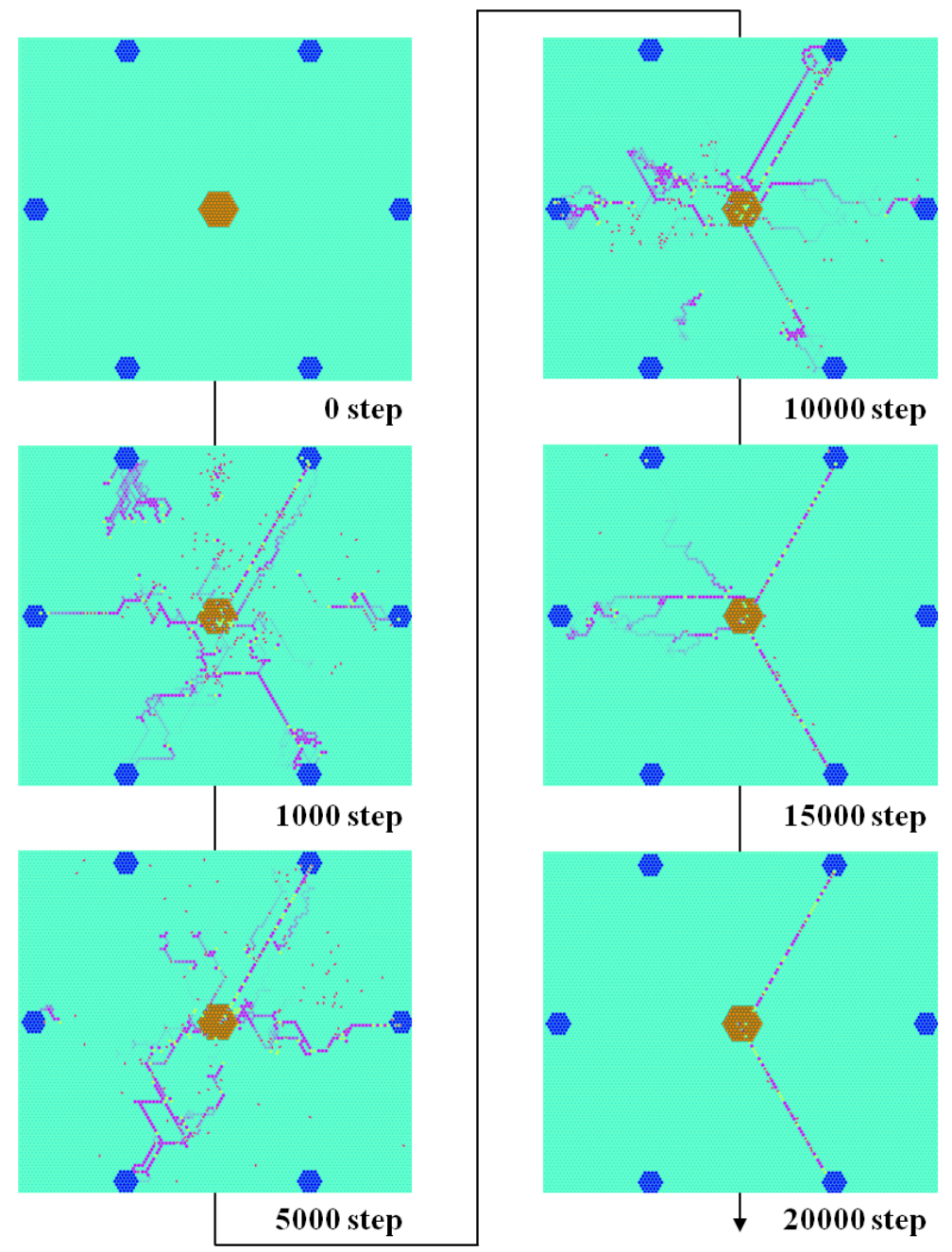

Fig. 4. Simulation of the formation of stable pheromone trails (purple lines) by the colony of 600 oriented ants. 
the histogram of the number of formed pheromone trails by repeating the simulation 50 times. It seems that there is a strong tendency to form pheromone trails between the nest and only two of the six food sources.

By the way, these results are sensitive to some parameter values in Table I. In particular, the evaporation coefficient of pheromones has crucial influence for forming stable foraging trails. For example, stable pheromone trails tend not to be formed if the coefficient is too large (e.g., 10 times larger than the original choice), while the trail formation tends to be slow if the coefficient is too small. In the case of that the coefficient is 10 times smaller than the original, the average number of steps to form stable pheromone trails is about 5 times larger. Moreover, the number of the resulting pheromone trails tends to decrease when the threshold concentration of grand pheromones is too large. In the case of that the coefficient is 100 times larger than the original, there is a extremely strong tendency to form pheromone trails between the nest and only one of the six food sources.

Next, we investigate the effect of changing the density of the oriented ant colony in the field. Figure 5(b-e) shows the histograms of the number of pheromone trails formed in 50 simulation runs, with 1500, 3000, 6000, and 9000 ants. From Fig. 5, we verify that the average number of resulting pheromone trails increases with the number of oriented ants. There is a strong tendency to form pheromone trails between the nest and all six food sources with 9000 oriented ants. Moreover, Fig. 6 shows the relation between the number of steps to converge the foraging behavior and the density of

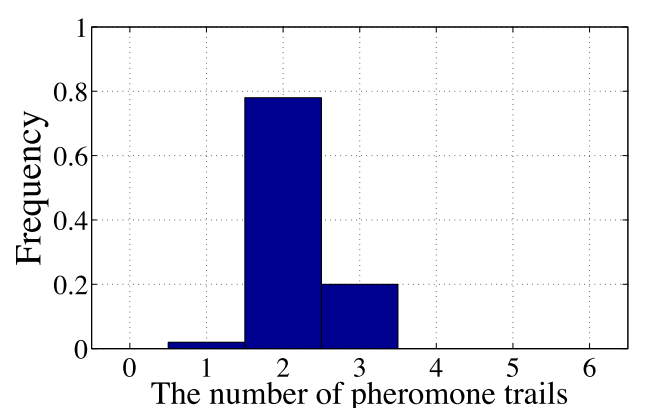

(a) 600 oriented ants

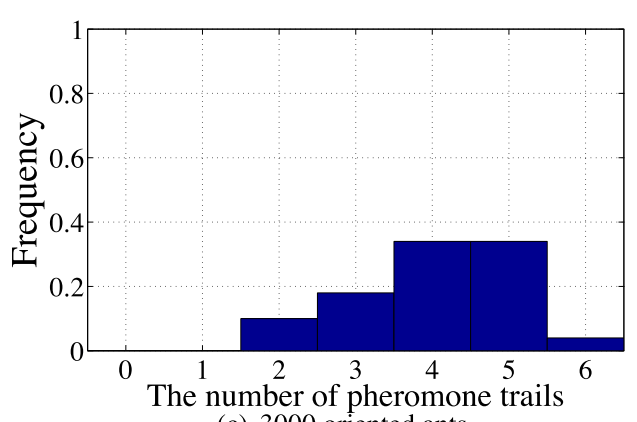

(c) 3000 oriented ants

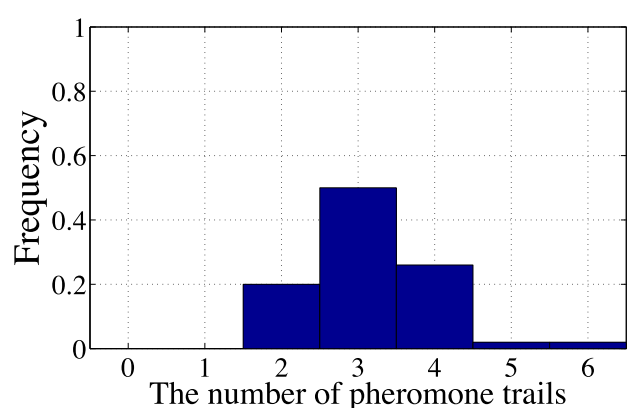

(b) 1500 oriented ants

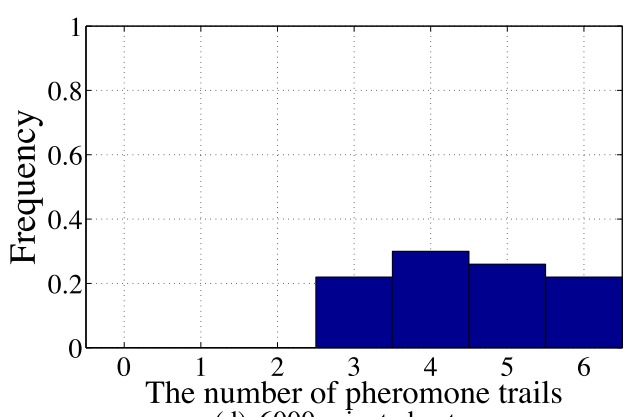

(d) 6000 oriented ants

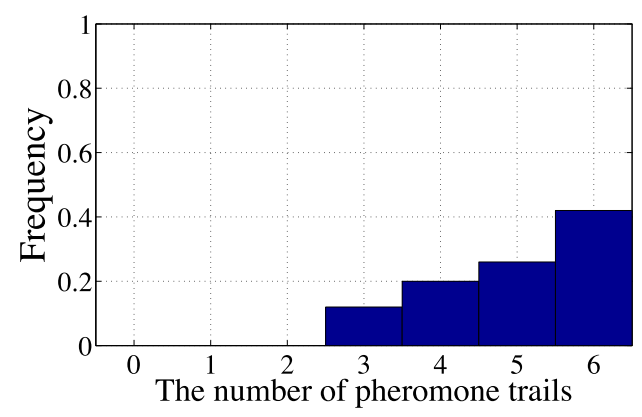

(e) 9000 oriented ants

Fig. 5. The relation between the number of pheromone trails formed and the density of oriented ants in the field. 


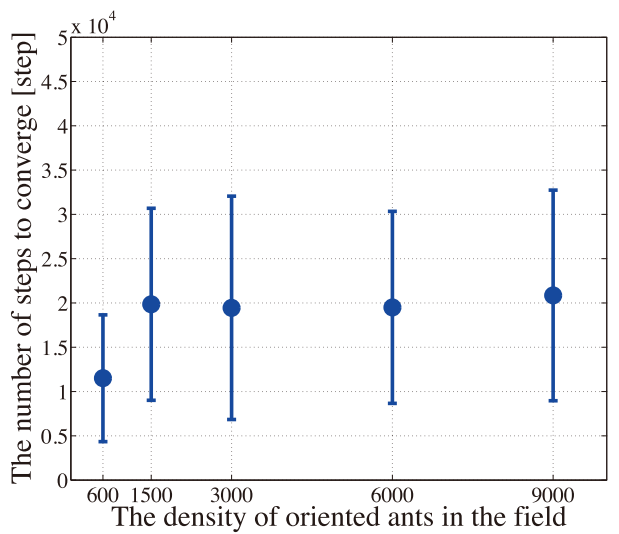

Fig. 6. The relation between the number of steps to converge the foraging behavior and the density of oriented ants in the field.

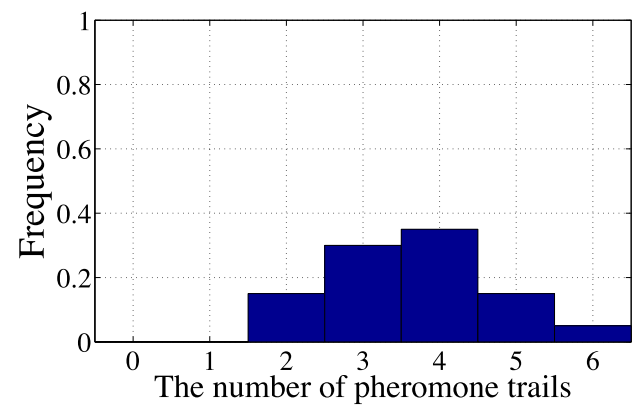

Fig. 7. The histogram of the number of pheromone trails formed by the mixed colony of 300 oriented ants and 300 random ants.

oriented ants in the field. Data are means \pm standard deviations. From Fig. 6 , we verify that the average number of steps for the convergence of the foraging behavior with 1500 ants is larger than that with 600 ants, but that with 1500 ants is almost as large as that with more than 3000 ants.

\subsection{Distributed transportation by a colony of heterogeneous agents}

We now discuss distributed transportation by a mixed colony of oriented ants and random ants, which follow different behavior procedures. The sum of the number of ants in the colony is fixed at 600 . First, we performed simulations on the distributed transportation by the mixed colony of 300 oriented ants and 300 random ants. Figure 7 shows the histogram of the number of pheromone trails formed for 50 runs of this simulation. Figure 7 shows that this mixed colony has a tendency to form a larger number of pheromone trails than the colony of only oriented ants, and they are likely to form four or three pheromone trails. In Fig. 8, we show the snapshot of two simulation results which this mixed colony formed three and four pheromone trails.

Next, several simulations were carried out by changing the density of the oriented ants and random ants. Figure 9(a) shows the average number of pheromone trails formed when the density of random ants in the colony changed. Figure 9(a) indicates that the stable pheromone trails are not formed by the colony of random ants only, and also indicates that a small number of random ants help to form pheromone trails between the nest and additional food sources compared to a colony of only oriented ants. There is a weak tendency to form pheromone trails between the nest and all six food sources, even if random ants are mixed in the colony of oriented ants. Figure 9(b) also shows the average of ratio of object transportation at the different densities of random ants. It appears that the ratio of object transportation decreases monotonically as the number of random ants increases.

\section{Distributed control of the number of pheromone trails based on local information}

As the previous sections show, oriented ants tend to form stable pheromone trails between their nest 


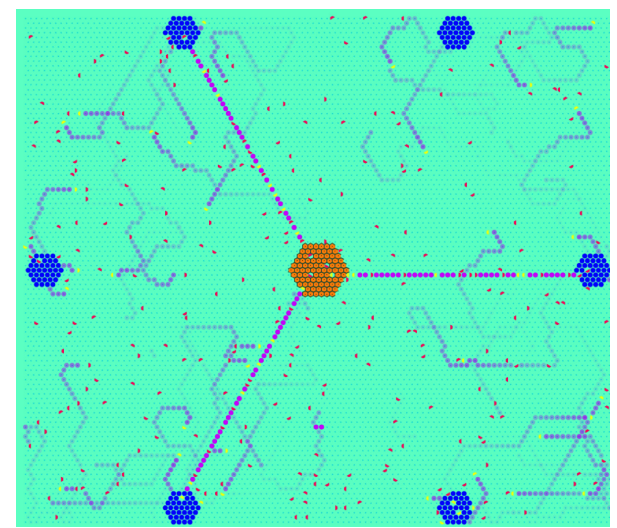

(a) Formation of three pheromone trails.

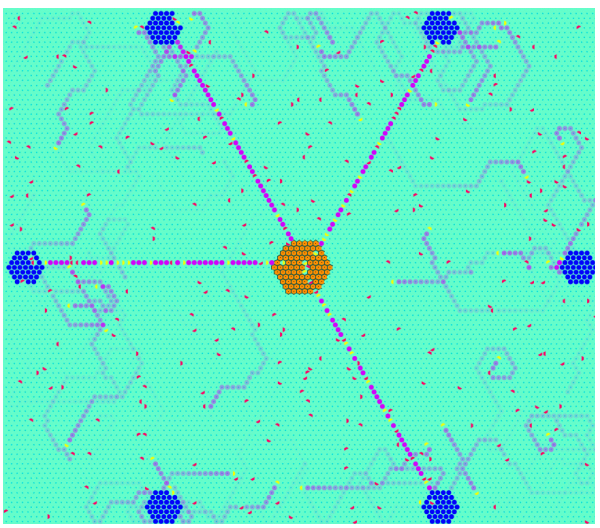

(b) Formation of four pheromone trails.

Fig. 8. Formation of pheromone trails (purple lines) by the mixed colony of 300 oriented ants and 300 random ants.

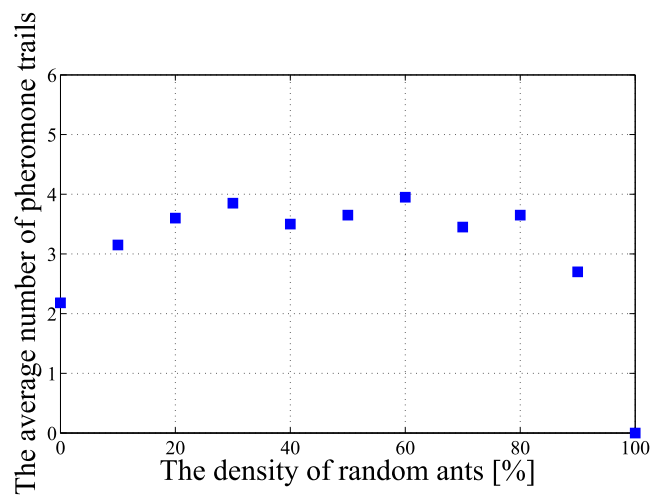

(a) The relation between the average number of pheromone trails (b) The relation between the ratio of transportation and the density and the density of random ants.

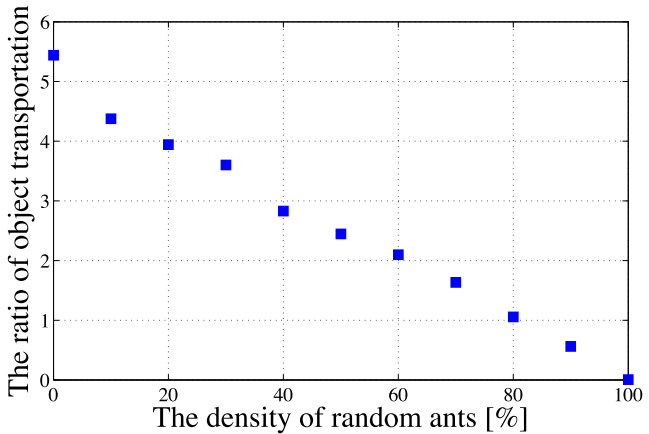
of random ants.

Fig. 9. Varying densities of heterogeneous agents.

and food sources, and random ants tend to explore new food sources. Here, let us consider how to form pheromone trails to all food sources from the nest. In order to exploit new food sources, random ants should be mixed into the colony. Next, oriented ants should be mixed into the colony so as to form stable pheromone trails between the nest and newly discovered food sources. Moreover, oriented ants tend to swarm to stable pheromone trails as shown in Fig. 4. In order to start utilizing other food sources at this time, random ants should be mixed into the colony again. The number of ants in the colony, however, is limited. In order to form pheromone trails between their nest and all food sources, we propose a new autonomous algorithm as follows: agents autonomously switch between oriented ant's behavior and random ant's behavior, based on the crowding of the current pheromone trail. However, the level of crowding of the current pheromone trail is global information.

We discuss how to estimate the crowdedness of the current pheromone trail. Pheromones on the trail thicken as the pheromone trail gets crowded. This indicates that the crowdedness of the pheromone trail - global information - can be estimated from the concentration of pheromones on the trail, which is local information. From the above considerations, we propose a distributed control method for the number of pheromone trails, by estimating the crowdedness from local concentration of pheromones around each ant. Every ant autonomously changes its behavior from oriented to random or vice versa, based on the concentration of pheromones perceived locally.

Every ant stores a memory for $L$ steps that records the concentration of locally perceived air pheromones. Based on this memory, every ant changes its behavior from oriented to random when the average of the concentration of air pheromones for $L$ steps passes a pre-determined threshold, and vice versa when the concentration drops under a pre-determined threshold, as shown in Fig. 10. In Fig. 10, $P_{L}$ denotes the average of the concentration of air pheromones locally perceived by each ant 


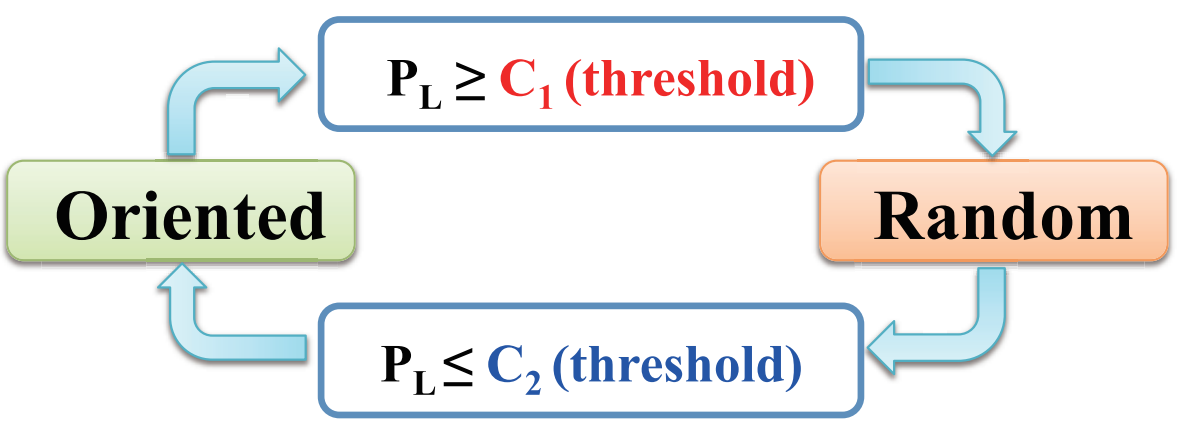

Fig. 10. Switching between the oriented behavior and the random one.
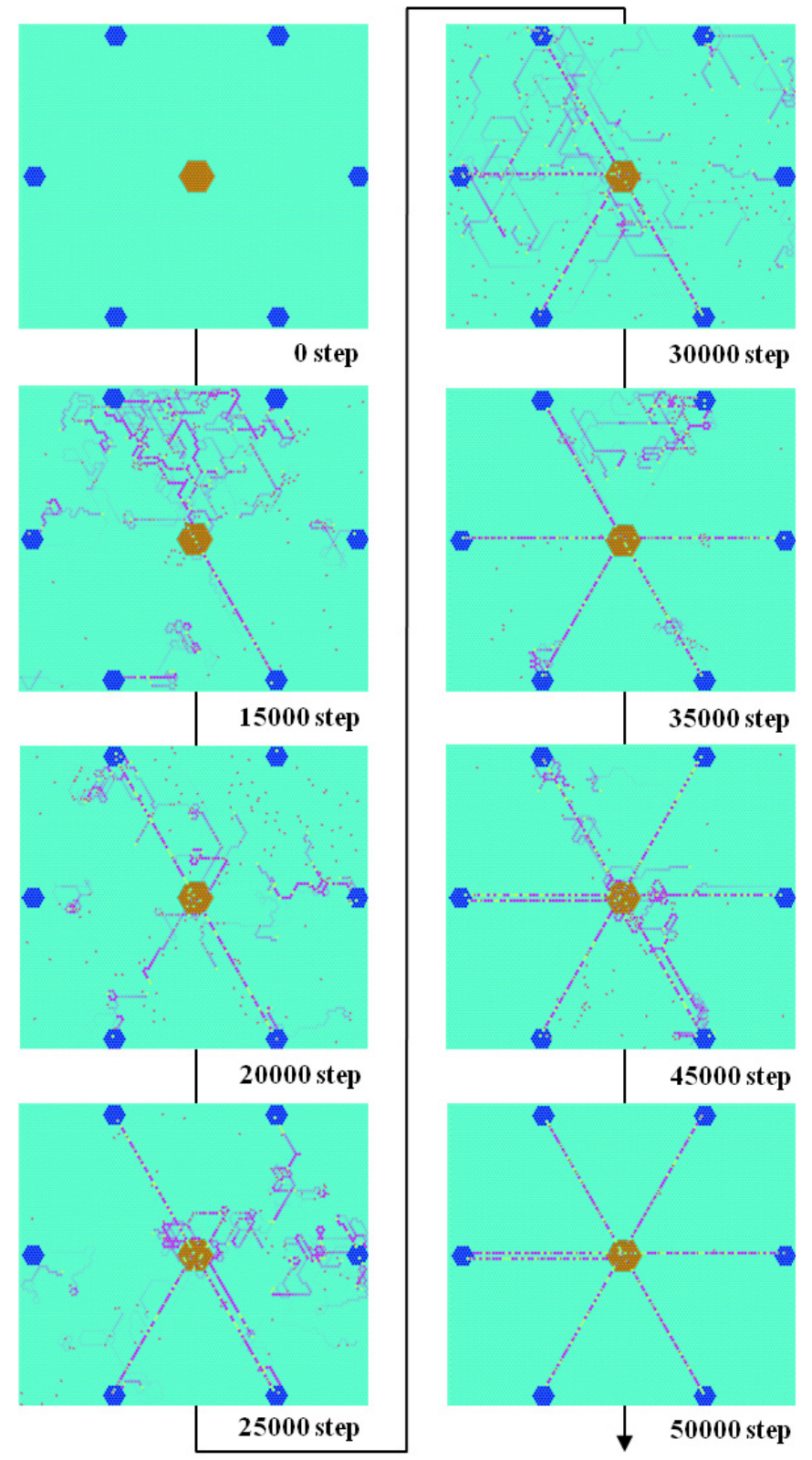

Fig. 11. Formation of six pheromone trails (purple lines) by 600 autonomous switching agents.

for $L$ steps. Here, three parameters are required for each ant, as described below:

- $C_{1}$ : the threshold of the concentration of air pheromones for changing ant's own behavior procedure from oriented ant's procedure to random ant's procedure. 


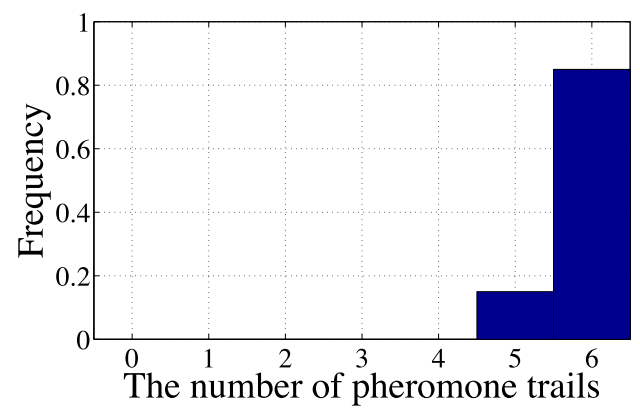

Fig. 12. The histogram of the number of pheromone trails formed by 600 autonomous switching agents.

- $C_{2}$ : the threshold of the concentration of air pheromones for changing ant's own behavior procedure from random ant's procedure to oriented ant's procedure.

- $L$ : the number of steps stored in the ant's memory

Now, we set three parameters to $C_{1}=500, C_{2}=350$ and $L=500$. The maximum amount of pheromones that every ant emits in one step is set to 100 .

We examine the effectiveness of the proposed control method by analyzing distributed transportation by the colony of 600 autonomous switching agents. Figure 11 shows one of the simulation results. We verify that the ants gradually form pheromone trails to all six food sources. Figure 12 shows the histogram of the number of pheromone trails formed over 50 simulation runs. There is a very strong tendency to form pheromone trails between the nest and all six food sources; pheromone trails are formed between the nest and at least five food sources in every run. Moreover, the average of the ratio of object transportation is 5.67 - close to that of the colony of only oriented ants (see Fig. 9(b)). From these simulations, we verify the effectiveness of proposed distributed control method for the number of pheromone trails.

\section{Conclusions and Future Work}

In this work we have focused on ant foraging behavior, using pheromones as one example of generating global patterns by autonomous mobile agents, and constructed a discrete event model of distributed transportation by the colony of ant agents. We then analyzed the distributed transportation by the colony of homogeneous or heterogeneous agents, using the number of pheromone trails formed between the ants' nest and food sources, and the ratio of object transportation as evaluation indices. Moreover, we proposed a distributed control method for the number of pheromone trails formed based on local pheromone information and verified the effectiveness of the proposed method in several simulations.

We are currently working on analyzing systematically this research by using evolutionary game theory. Furthermore, behavior of agents in this research will be coded on the basis of the logical operations. We aim to analyze distributed transportation with autonomous agents comprehensively, as an elementary cellular automaton in future work.

\section{Acknowledgments}

This research is partially supported by Grant-in-Aid for JSPS Fellows Grant Number 257750.

\section{References}

[1] K. Kawabata, H. Aonuma, K. Hosoda, and J. Xue, "Development of a cricket interaction system utilizing mobile robot for behavioral data collection," in Proc. International Conference on Robotics and Biomimetics (ROBIO 2012), pp. 1615-1620, 2012.

[2] M. Hansell, Built by Animals: The Natural History of Animal Architecture, Oxford University Press, 2009.

[3] B. Hölldobler and E.O. Wilson, The Ants, Belknap Press of Harvard University Press, 1990. 
[4] R. Yamaoka and T. Akino, "Ecological importance of cuticular hydrocarbon secreted from the tarsus of ants," in Les Insectes Sociaux, Published by Univ. Paris-Nord, pp. 222, 1994.

[5] B. Hölldobler and E.O. Wilson, Journey to the Ants: A Story of Scientific Exploration, Belknap Press of Harvard University Press, 1994.

[6] G. Caprari and R. Siegwart, "Mobile micro-robots ready to use: Alice," in Proc. IEEE/RSJ International Conference on Intelligent Robots and Systems, pp. 3295-3300, 2005.

[7] T.H. Labella, M. Dorigo, and J.-L. Deneubourg, "Division of labour in a group of robots inspired by ants' foraging behavior," in ACM Transactions on Autonomous and Adaptive Systems, vol. 1, no. 1 , pp. 4-25, 2006.

[8] S. Garnier, F. Tache, M. Combe, A. Grimal, and G. Theraulaz, "Alice in pheromone land: An experimental setup for the Study of Ant-like robots," in Proc. IEEE Swarm Intelligence Symposium, SIS 200\%, pp. 37-44, 2007.

[9] R. Mayet, J. Roberz, T. Schmickl, and K. Crailsheim, "Antbots: A feasible visual emulation of pheromone trails for swarm robots," in Proc. "Tth International Conference on Swarm Intelligence (ANTS 2010), pp. 84-94, 2010.

[10] R. Fujisawa, H. Imamura, and F. Matsuno, "Cooperative transportation on Swarm Robot Using Pheromone Communication," in Springer Tracts in Advanced Robotics - The 10th International Symposium, pp. 559-570, 2012.

[11] M. Dorigo, "Optimization, learning and natural algorithms," in Ph.D. Thesis, Politecnico di Milano, Italy, 1992.

[12] M. Dorigo, G.D. Caro, and L.M. Gambardella, "Ant algorithms for discrete optimization," in Artificial life, vol. 5, no. 2, pp. 137-172, 1999.

[13] S. Wolfram, Cellular Automata and Complexity: Collected Papers, Westview Press, 1994.

[14] J.L. Schiff, Cellular Automata : A Discrete View of the World, Wiley-Interscience, 2007.

[15] M. Ishikawa, "On spatial discretization of mobile robot systems and related control problems," in Proc. SICE 40th Symposium on Control Theory, 2011.

[16] T. Kita, M. Ishikawa, and K. Osuka, "On discrete-valued modeling of nonholonomic mobile robot systems," in Proc. International Conference on Robotics and Biomimetics (ROBIO 2012), pp. 2024-2031, 2012.

[17] M. Nakamura and K. Kurumarani, "A method for designing ant colony models and two examples of its application," in Advances in Artificial Life, Lecture Notes in Computer Science, vol. 3630, pp. 530-539, 2005.

[18] M. Nakamura, "Regulation mechanism of task-allocation and formation mechanism of ants' distribution pattern in collective behavior of ant colony models," in Soft Computing as Transdisciplinary Science and Technology, Advances in Soft Computing, vol. 29, pp. 937-948, 2005.

[19] R.P. Evershed, E.D. Morgan, and M.C. Cammaerts, "3-ethyl-2,5-dimethylpyrazine, the trail pheromone from the venom gland of eight species of Myrmica ants," in Insect Biochemistry, vol. 12 , pp. 383-395, 1982.

[20] J. Traniello, "Olfaction and chemical communication," in Tested studies for laboratory teaching, vol. 18, pp. 167-185, 1996.

[21] R. Beckers, J.L. Deneubourg, and S. Goss, "Trails and U-turns in the selection of a path by the Ant Lasius niger," in Journal of Theoretical Biology, vol. 159, pp. 397-415, 1992.

[22] S. Camazine, J.L. Deneubourg, N.R. Franks, J. Sneyd, G. Theraulaz, and E. Bonabeau , SelfOrganization in Biological Systems, Princeton University Press, 2001.

[23] R. Wehner and M. Müller, "The significance of direct sunlight and polarized skylight in the ant's celestial system of navigation," in Proc. National Academy of Sciences USA, vol. 103, no. 33, pp. $12575-12579,2006$. 